\title{
ORIGINAL ARTICLE \\ Acute spinal cord injury could cause activation of autophagy in dorsal root ganglia
}

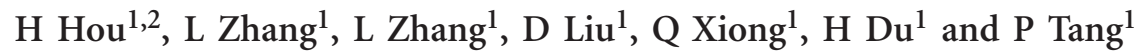

Objectives: Dorsal root ganglia (DRGs) have an important role in the peripheral mechanism of sensation by primary afferent neurons, which are widely used to research the processes of cell death, axonal regeneration, signal transmission of growth factors and the mechanism of pain.

Methods: In the present study, we investigated the activation of autophagy in DRGs in a rat model of acute spinal cord injury at different time points.

Results: Expression of microtubule-associated protein light chain 3, a marker of autophagy was increased after $8 \mathrm{~h}$ in DRGs, peaked after 3 days, and then gradually decreased after 7 days. Furthermore, the toluidine blue staining has proven that after acute spinal cord injury, the myelin sheathes of DRGs undergo histopathological changes over time, with axonal swellings, disorderly arrangement and uneven distribution.

Conclusion: Potential treatment aimed at recovery of behavioral locomotor and sensory perception should target the process of autophagy in DRGs.

Spinal Cord (2013) 51, 679-682; doi:10.1038/sc.2013.52; published online 2 July 2013

Keywords: autophagy; dorsal root ganglia (DRGs); acute spinal cord injury (SCl); LC3

\section{INTRODUCTION}

Dorsal root ganglia (DRGs) contain glial cells and the bodies of primary sensory neurons, which carry somatosensory information from the soma to the central nervous system, ${ }^{1}$ and are pivotal in neuronal transmission between the peripheral nervous system and the central nervous system. ${ }^{2}$ These neurons have bipolar morphology, comprising an undifferentiated terminal with the synaptic terminal entering the dorsal horn of the spinal cord. ${ }^{3}$ In the membrane of DRG neurons, many kinds of ion channels and receptors are expressed; these can change in response to nerve injury and can be involved in various physiological and pathological pathways. ${ }^{4}$

Autophagy is an active mechanism for degrading damaged organelles and unwanted or long-lived proteins in the autophagosome-lysosome pathway. It was first discovered and described in conditions of starvation; ${ }^{5}$ when there is a shortage of nutrients, the process of autophagy is activated. A double membrane is formed around cellular substances, creating the autophagosome, which then fuses with a lysosome, following which these organelles and proteins are degraded and recycled back to the cell itself as amino and fatty acids to enable cell survival. ${ }^{6}$ Autophagy is a part of various biochemical pathways and diseases, including tumor suppression, ${ }^{7}$ removal of toxic agents, ${ }^{8}$ infection, ${ }^{9}$ and heart disease. ${ }^{10}$ Recently, the role of autophagy in the nervous system has been assessed, especially in relation to the pathogenesis of Alzheimer's disease, ${ }^{11}$ Parkinson's disease $^{12}$ and amyotrophic lateral sclerosis ${ }^{13,14}$ in the peripheral nervous system.

The microtubule-associated protein light chain 3 (LC3) appears to have a crucial role in the formation of autophagosomes. LC3 is lipidated upon activation of autophagy (LC3I is the unlipidated form and LC3-II is the lipidated form). LC3 is initially synthesized in an unprocessed form, pro-LC3, which is converted into a proteolytically processed form, LC3-I, which lacks amino acids from the C terminus, and LC3-I is finally modified into the phosphatidylethanolamine (PE)-conjugated form, LC3-II. ${ }^{6}$ Previous studies used the upregulation of LC3 to investigate the induction of autophagy in several disease models. ${ }^{15-17}$

In the present study, we focused on the process of autophagy in the DRGs after acute spinal cord injury (SCI), and describe the relationship between autophagy and the DRGs, which indicated a potential therapeutic target to enable recovery of locomotor and sensory functions.

\section{MATERIALS AND METHODS}

\section{Animals and procedures}

All animal procedures were carried out in compliance of the guidelines for scientific animal procedures approved by our institution's ethics committee. In total, 36 male Sprague-Dawley rats weighing $220-250$ g were used for the study. The rats were randomly divided into six groups of six animals each, one control group and five experimental groups. The rats were housed, three or four to a cage, 8 days before the experiments began, on a 12-h light/dark cycle with the temperature kept at $25^{\circ} \mathrm{C}$.

For the experimental procedure, animals were anesthetized with $10 \%$ pentobarbital sodium $300 \mathrm{mg} \mathrm{kg}^{-1}$ administered intraperitoneally. The temperature of the animals was recorded and maintained at $37^{\circ} \mathrm{C}$ with a heating pad during the procedures. A laminectomy was performed with the aid of a dissecting microscope to expose the spinal cord at the level of T9-T10. Following the midline of the cord, a dorsal hemisection (at the right side) was completed, and any residual fibers removed. The experimental groups were performed at $8 \mathrm{~h}$ and $1,3,7$ and 21 days after SCI. The control group

${ }^{1}$ Department of Orthopaedics, Chinese PLA General Hospital, Beijing, China and ${ }^{2}$ Medical College, Nankai University, Tianjin, China

Correspondence: Dr P Tang, Department of Orthopaedics, Chinese PLA General Hospital, Beijing Haidian District Fuxing Road No. 28, Beijing 100853, China.

E-mail: pftang301@126.com

Received 25 February 2013; revised 8 April 2013; accepted 16 April 2013; published online 2 July 2013 
underwent the same procedure without hemisection. The bladder and bowel of each rat was manipulated twice a day after surgery until recovery of the self-voiding function occurred.

\section{Immnohistochemical staining}

At each time point, three animals in each group were transcardially perfused with phosphate-buffered saline (PBS), followed by $0.1 \mathrm{~m}$ phosphate buffer containing $4 \%$ paraformaldehyde. DRGs are generally located on the intervertebral canal, outside of spinal dural sheath. ${ }^{18}$ In represent research, the T8-L5 levels of the spine were removed and the DRGs in the intervertebral foramen on the injured side were removed one by one into the fixative. These DRGs were then post-fixed in PBS containing 30\% sucrose until they precipitated to the bottom of the container. The fixed tissue was frozen and $20 \mu \mathrm{m}$ sections were cut on a cryostat, then mounted on slides.

To investigate the induction of autophagy in the DRGs after acute SCI, immunohistochemical staining was performed. Sections were washed with PBS three times for $5 \mathrm{~min}$ each, and then boiled in $0.1 \%$ tri-sodium citrate for $15 \mathrm{~min}$. Following this, sections were incubated with blocking reagent (5\% goat serum diluted in $0.3 \%$ PBS containing Triton X-100) for $1 \mathrm{~h}$ at room temperature, followed by overnight incubation at $4{ }^{\circ} \mathrm{C}$ with the primary antibody (rabbit polyclonal anti-LC3 Sigma-Aldrich, St Louis, MO, USA), ${ }^{19-21}$ which was diluted 1:200 in PBS containing 5\% goat serum and $0.1 \% \mathrm{NaN}_{3}$. The secondary antibody was fluorescein isothiocyanate-conjugated goat antirabbit IgG (Abcam, Cambridge, MA, USA) diluted in PBS.

\section{Toluidine blue staining}

The DRGs sections were stained with toluidine blue to investigate the degree of demyelination in the different groups. The remaining three animals from each group were transcardially perfused with PBS and $2.5 \%$ glutaradehyde at the relevant time points $8 \mathrm{~h}$, and 1, 3, 7 and 21 days. The DRGs at the levels of T8-L5 were removed and then placed in $2.5 \%$ glutaradehyde fixative. The tissue was then cut into semi-thin sections (about $0.6 \mu \mathrm{m}$ ), which were stained with toluidine blue.

\section{Statistics}

All images were analyzed using Image-Pro Plus software (Media Cybernatics, Inc., Silver Spring, MD, USA). Data are reported as Means \pm s.d. The number of cells with punctuate LC3 fluorescence was counted and the percentages of positive cells were calculated in 50 randomly chosen slices per animal and averaged, then used for quantitative analysis. Percentage of demyelination was done and analyzed as the same. Significant differences between animals at each time points were assessed by ANOVA, using SPSS17.0 software (SPSS Inc., Chicago, IL, USA), with $P<0.05$ considered statistically significant.

\section{RESULTS}

\section{LC3 is upregulated in DRG after acute SCI}

Cells positive for LC3 were sparse in the DRGs of control animals (Figure 1a). At $8 \mathrm{~h}$ after injury, the number of LC3-positive cells was increased, peaking at 3 days, and then decreasing from 7 days after acute SCI (Figure 1a). These results indicate that the process of autophagy was activated in these DRGs after ipsilateral SCI (Figure 1b).

\section{Tissues in the DRG after acute SCI were demyelinated and had a disorderly arrangement}

In the control group, the sections contained myelin sheaths of different diameters in an orderly arrangement and evenly distributed around the nerves (Figure 2). Demyelination started to appear $8 \mathrm{~h}$ after injury, and lasted until 3 days, with a disorderly arrangement and irregular distribution, accompanied by many swellings and neardisruption. After 7 days, these pathological changes began to improve, which coincided with the recovery of blood flow and nutrition.
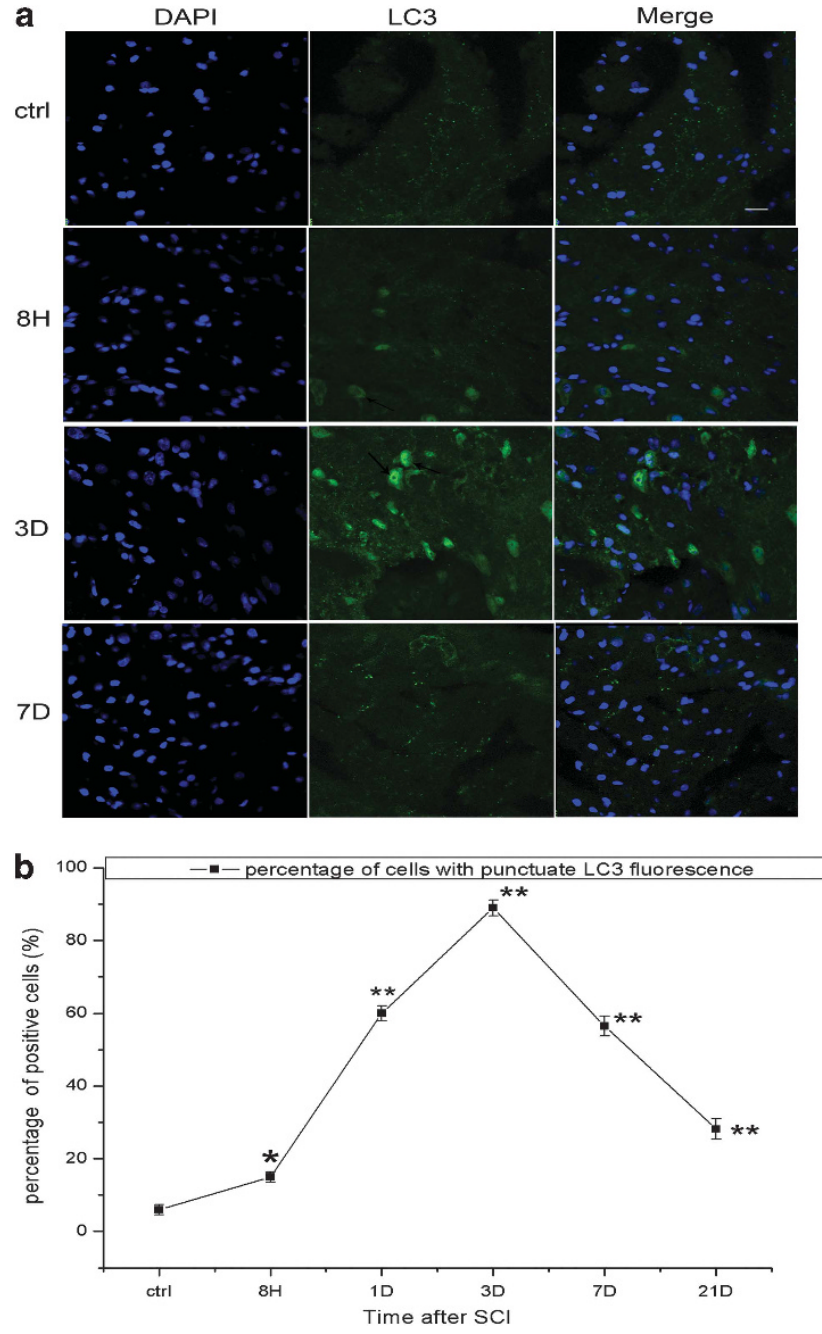

Figure $1 \mathrm{Immunohistochemical} \mathrm{staining} \mathrm{of} \mathrm{LC3} \mathrm{in} \mathrm{the} \mathrm{DRG} \mathrm{after} \mathrm{acute} \mathrm{SCI.}$ (a) In the control group, LC3-positive cells were sparse, whereas in the injured group, the population of LC3-positive cells increased after $8 \mathrm{~h}$, peaked at 3 days, and then gradually decreased at 7 days. (Arrows show the positive cells with LC3.) (b) Analysis of percentage of cells in DRGs with punctuate LC3 fluorescence. ${ }^{*} P<0.05,{ }^{*} P<0.01$. Scale bar $=20 \mu \mathrm{m}$.

\section{DISCUSSION}

DRGs contain neurons with bipolar morphology, which have an important role in transmission within the central nervous system and peripheral nervous system. ${ }^{22}$ Recent studies have focused on the role of the DRGs in the mechanisms of pain sensitivity and neurological pain. $^{23-25}$ In one type of peripheral neuropathy, acquired sensory ganglionopathy, the sensory nerve cell bodies in the DRGs were damaged, resulting in sensory loss in the large fibers. ${ }^{26}$ Most of the distal axonpathies have been shown to display simultaneous distal degeneration of both the peripheral and central axons of DRGs, a condition termed central-peripheral distal axonopathy, whereas others display only selective distal degeneration of the peripheral axons. ${ }^{27}$ In many chronic pain managements, DRGs had very important roles. ${ }^{28}$

Autophagy is a cellular mechanism of self-cycling for survival, which is also called type 2 cell death or autophagic cell death. The process of autophagy is involved in many diseases of the nervous system and in nerve injuries. In Alzheimer's disease, overactivation of autophagy has been shown to slow down the symptoms and help 

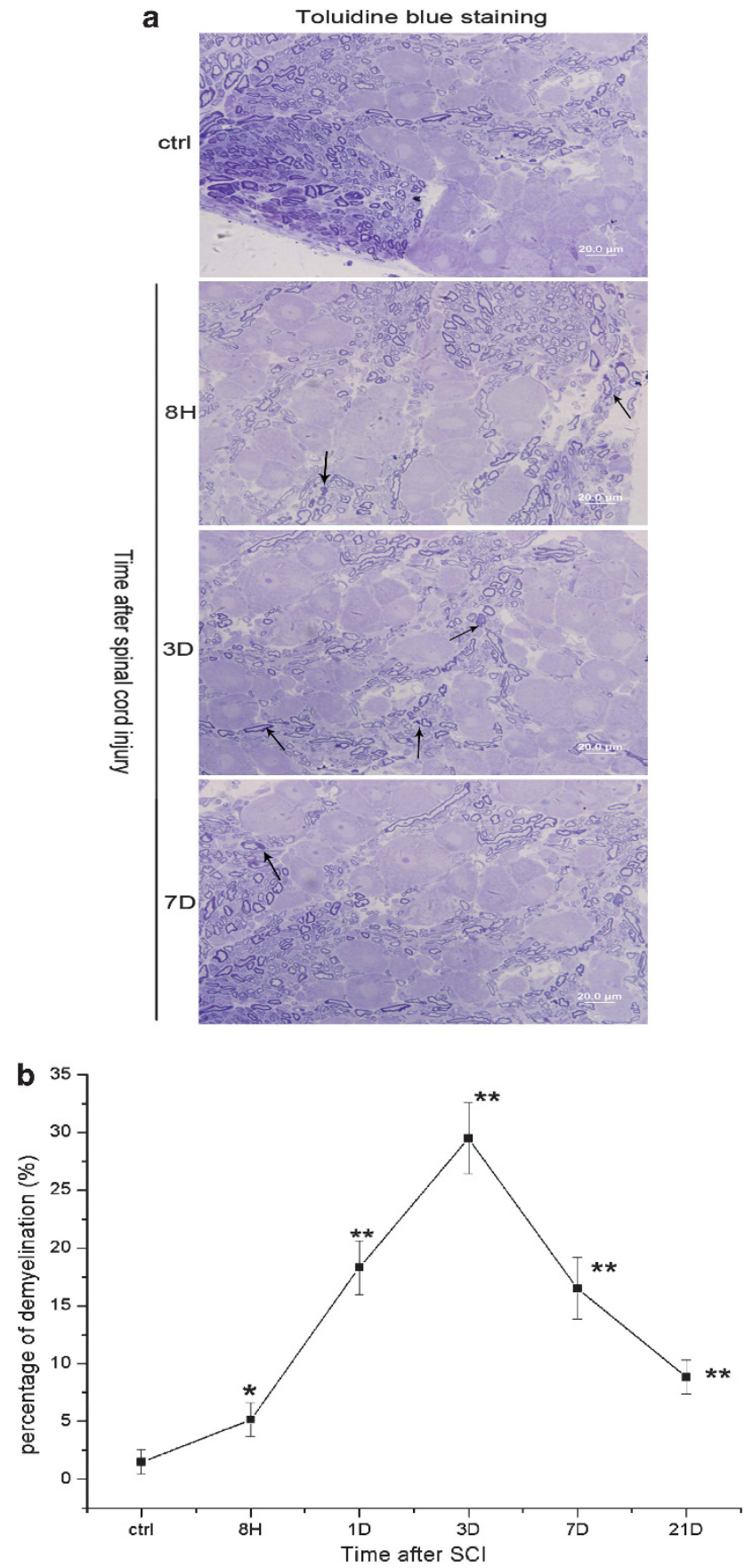

Figure 2 Toluidine blue staining of DRG in controls and at each time point after acute SCl. (a) In the control group, the myelin sheaths have a normal shape and diameter, whereas in the injured rats, the myelin sheaths have a disorderly arrangement and uneven distribution. The demyelination peaked on 3 days after injury. Then the pathological changes began to improve after 7 days, as a result of recovery of blood flow and nerve regeneration. (Arrows show the disorderly arrangement of demyelinated sheaths.) (b)Analysis of percentage of demyelination in DRGs after $\mathrm{SCl} .{ }^{*} P<0.05,{ }^{* *} P<0.01$. Scale bar $=20 \mu \mathrm{m}$.

memory recovery in animal models. ${ }^{29,30}$ In other acute brain injuries, autophagy may have a different role, depending on the models and the timing of observation. In the model of closed head injury, overexpression of the protein Beclin1 was able to remove injured cells and reduce damage to other cells, indicating a potential protective role for autophagy. However, in the model of neonatal cerebral ischemia, inhibition of autophagy decreased the area of ischemic penumbra and neuroprotective efficacy. ${ }^{31}$

In the current study, we detected activation of autophagy in DRGs after acute SCI. Expression of LC3 was increased in DRGs after injury, and changed over time, appearing at $8 \mathrm{~h}$, peaking at 3 days and gradually decreasing from 7 days. Our results demonstrate the activation of autophagy in DRGs after SCI, and DRGs might participate in the process of axonal degeneration and regeneration after SCI.

The myelin sheath is a layer of adipose tissue, wrapped in specific neuronal axons, with an insulating intermediate layer, which improves the conduction velocity of nerve impulses and helps to protect the axons. Rangaraju and Notterpek ${ }^{32}$ reported that when activation of autophagy was enhanced by rapamycin, Schwann cells isolated from normal mice could maintain their myelinating phenotype in explant cultures of DRG neurons. Along with the morphological changes, the processing and trafficking of peripheral myelin protein 22 also improved, and this was associated with an increase in the length and abundance of myelin internodes. ${ }^{33}$ However, the potential mechanism between autophagy and myelination has not yet been explained, although it may involve the endocytic or other pathways. What is the definite role of autophagy in demyelination and remyelination in DRGs after SCI? Rapamycin, an enhancer of authophagy, and 3-methyladenine, an inhibitor of autophagy, will be used in further studies to try to explain this process.

In conclusion, we report activation of autophagy in the DRGs after SCI, the first such report, to our knowledge. This indicates that autophagy may be involved in the pathological changes occurring after SCI and may have a role in demyelination and recovery of function after SCI. Further investigation into the role of autophagy in the DRGs after SCI is needed, and it may indicate potential for treatment of SCI in the future.

\section{DATA ARCHIVING}

There were no data to deposit.

\section{CONFLICT OF INTEREST}

The authors declare no conflict of interest.

\section{ACKNOWLEDGEMENTS}

This research was supported by the Natural Science Foundation of China (Grant no. 30973068) and the General Projects of the Twelve-Fifth Scientific Plan in Army Medical Science and Technology (Grant no. CWS11J101).

1 Rozas JL. Metabotropic actions of kainate receptors in dorsal root ganglion cells. Adv Exp Med Biol 2011; 717: 69-80.

2 Miller RJ, Jung $\mathrm{H}$, Bhangoo SK, White FA. Cytokine and chemokine regulation of sensory neuron function. Handb Exp Pharmacol 2009; 194: 417-449.

3 Schaeffer V, Meyer L, Patte-Mensah C, Mensah-Nyagan AG. Progress in dorsal root ganglion neurosteroidogenic activity: basic evidence and pathophysiological correlation. Prog Neurobiol 2010; 92: 33-41.

4 Wang W, Gu J, Li YQ, Tao YX. Are voltage-gated sodium channels on the dorsal root ganglion involved in the development of neuropathic pain? Mol Pain 2011; 7: 16.

5 Trump BF, Berezesky IK, Phelps PC. Sodium and calcium regulation and the role of the cytoskeleton in the pathogenesis of disease: a review and hypothesis. Scan Electron Microsc 1981;(Pt 2): 435-454, 434.

6 Mizushima N, Yoshimori T, Levine B. Methods in mammalian autophagy research. Cell 2010; 140: 313-326.

7 Beau I, Mehrpour M, Codogno P. Autophagosomes and human diseases. Int J Biochem Cell Biol 2011; 43: 460-464

8 Mijaljica D, Prescott M, Devenish RJ. Autophagy in disease. Methods Mol Biol 2010; 648: 79-92. 
9 Eskelinen EL, Saftig P. Autophagy: a lysosomal degradation pathway with a central role in health and disease. Biochim Biophys Acta 2009; 1793: 664-673.

10 Bao XH, Naomoto Y, Hao HF, Watanabe N, Sakurama K, Noma K et al. Autophagy: can it become a potential therapeutic target? Int J Mol Med 2010; 25: 493-503.

11 Cheung ZH, Ip NY. Autophagy deregulation in neurodegenerative diseases-recent advances and future perspectives. J Neurochem 2011; 118: 317-325.

12 Hakimi M, Selvanantham T, Swinton E, Padmore RF, Tong Y, Kabbach G et al. Parkinson's disease-linked LRRK2 is expressed in circulating and tissue immune cells and upregulated following recognition of microbial structures. J Neural Transm 2011; 118: 795-808.

13 Ferrucci M, Fulceri F, Toti L, Soldani P, Siciliano G, Paparelli A et al. Protein clearing pathways in ALS. Arch Ital Biol 2011; 149: 121-149.

14 Zhang X, Li L, Chen S, Yang D, Wang Y, Zhang X et al. Rapamycin treatment augments motor neuron degeneration in SOD1(G93A) mouse model of amyotrophic lateral sclerosis. Autophagy 2011; 7: 412-425.

15 Mizushima N, Yamamoto A, Matsui M, Yoshimori T, Ohsumi Y. In vivo analysis of autophagy in response to nutrient starvation using transgenic mice expressing a fluorescent autophagosome marker. Mol Biol Cell 2004; 15: 1101-1111.

16 Feng R, Wang SY, Shi YH, Fan J, Yin XM. Delphinidin induces necrosis in hepatocellular carcinoma cells in the presence of 3-methyladenine, an autophagy inhibitor. J Agric Food Chem 2010; 58: 3957-3964.

17 Erlich S, Alexandrovich A, Shohami E, Pinkas-Kramarski R. Rapamycin is a neuroprotective treatment for traumatic brain injury. Neurobiol Dis 2007; 26: 86-93.

18 Tucker BA, Rahimtula M, Mearow KM. A procedure for selecting and culturing subpopulations of neurons from rat dorsal root ganglia using magnetic beads. Brain Res Brain Res Protoc 2005; 16: 50-57.

19 Berglund L, Bjorling E, Oksvold P, Fagerberg L, Asplund A, Szigyarto CA et al. A genecentric human protein Atlas for expression profiles based on antibodies. Mol Cell Proteomics 2008; 7: 2019-2027.

20 Funk KE, Mrak RE, Kuret J. Granulovacuolar degeneration (GVD) bodies of Alzheimer's disease (AD) resemble late-stage autophagic organelles. Neuropathol Appl Neurobiol 2011; 37: 295-306.

21 Ait-Mohamed O, Battisti V, Joliot V, Fritsch L, Pontis J, Medjkane S et al. Acetonic extract of Buxus sempervirens induces cell cycle arrest, apoptosis and autophagy in breast cancer cells. PLoS One 2011; 6: e24537.
22 de Groat WC, Yoshimura N. Changes in afferent activity after spinal cord injury. Neurourol Urodyn 2010; 29: 63-76.

23 Martinez-Lavin M, Solano C. Dorsal root ganglia, sodium channels, and fibromyalgia sympathetic pain. Med Hypotheses 2009; 72: 64-66.

24 Waxman SG. Nav1.7, its mutations, and the syndromes that they cause. Neurology 2007; 69: 505-507.

25 Bremner F, Smith S. Pupillographic findings in 39 consecutive cases of harlequin syndrome. J Neuroophthalmol 2008; 28: 171-177.

26 Sheikh SI, Amato AA. The dorsal root ganglion under attack: the acquired sensory ganglionopathies. Pract Neurol 2010; 10: 326-334.

27 Thomas PK. Selective vulnerability of the centrifugal and centripetal axons of primary sensory neurons. Muscle Nerve 1982; 5: S117-S121.

28 Fuentes R, Petersson P, Nicolelis MA. Restoration of locomotive function in Parkinson's disease by spinal cord stimulation: mechanistic approach. Eur J Neurosci 2010; 32 1100-1108.

29 Yang DS, Stavrides P, Mohan PS, Kaushik S, Kumar A, Ohno M et al. Therapeutic effects of remediating autophagy failure in a mouse model of Alzheimer disease by enhancing lysosomal proteolysis. Autophagy 2011; 7: 788-789.

30 Barnett A, Brewer GJ. Autophagy in aging and Alzheimer's disease: pathologic or protective? J Alzheimers Dis 2011; 25: 385-394.

31 Puyal J, Vaslin A, Mottier V, Clarke PG. Postischemic treatment of neonatal cerebral ischemia should target autophagy. Ann Neurol 2009; 66: 378-389.

32 Rangaraju S, Verrier JD, Madorsky I, Nicks J, Dunn WJ, Notterpek L. Rapamycin activates autophagy and improves myelination in explant cultures from neuropathic mice. J Neurosci 2010; 30: 11388-11397.

33 Rangaraju S, Notterpek L. Autophagy aids membrane expansion by neuropathic Schwann cells. Autophagy 2011; 7: 238-239.

(1) $(\Theta$ This work is licensed under a Creative Commons Attribution-NonCommercial-NoDerivs 3.0 Unported License. To view a copy of this license, visit http://creativecommons. org/licenses/by-nc-nd/3.0/ 